\title{
High resolution observation of 17P/ Holmes during the outburst event in 2007
}

\author{
M. T. Capria $^{1}$, G. Cremonese ${ }^{2}$, and M. C. De Sanctis ${ }^{1}$
}

1 INAF - IASF, Area Ricerca di Tor Vergata, Rome, Italy

e-mail: mariateresa.capria@iasf-roma.inaf.it

2 INAF - Padua Observatory, vicolo dell'Osservatorio 5, Padua, Italy

Received 16 December 2009 / Accepted 12 April 2010

ABSTRACT

\begin{abstract}
Aims. On October 24, 2007 the comet 17P/ Holmes underwent an outburst that was extraordinary in both duration and size. We attempted to determine the cause of the outburst by acquiring and analyzing high resolution spectra of the comet.

Methods. On two nights, October 25 and December 2, we acquired four high resolution spectra in the visible range at the Telescopio Nazionale Galileo in La Palma. The oxygen lines intensity ratio was estimated for the observation performed on October 25, and computed for the observation performed on December 2.

Results. Many emission lines were detected in the spectra, most of them attributable to $\mathrm{C}_{2}, \mathrm{NH}_{2}, \mathrm{CN}$ and $\mathrm{CH}$. The atomic $\mathrm{O}$ green line and one of the red doublet lines were detected in a spectrum acquired on October 25. All the three atomic $\mathrm{O}$ lines were detected in a spectrum acquired on December 2. The value obtained for the first night, $0.1 \pm 0.11$, implies that the $\mathrm{H}_{2} \mathrm{O}$ molecule was the main parent of the emission lines, while the value obtained for the second night, $0.3 \pm 0.1$, implies that the main parent was $\mathrm{CO}$ or $\mathrm{CO}_{2}$ instead than $\mathrm{H}_{2} \mathrm{O}$.

Conclusions. Our results imply that in the days around the outburst the water production was exceptionally high for that heliocentric distance. By December, however, the behavior of 17P/ Holmes had returned to that of a comet orbiting at 2.6 AU from the Sun, in which state the activity is probably sustained mostly by gases more volatile than water, such as $\mathrm{CO}$ and $\mathrm{CO}_{2}$.
\end{abstract}

Key words. comets: general - comets: individual: 17P/ Holmes

\section{Introduction: the extraordinary outburst of 17P/ Holmes}

Comet $17 \mathrm{P} /$ Holmes is a Jupiter-family comet that in late October 2007 underwent an outburst extraordinary in both duration and size (Green 2007). The orbital period of the comet is 6.9 years and the inclination is 19.11 degrees; the perihelion is reached at 2.05 AU from the Sun. 17P/ Holmes was discovered on November 6, 1892 by Holmes, during an extraordinary brightness increase, followed by another similar event on January 16, 1893. On October 24, 2007, 173 days after the last perihelion passage, the comet underwent a similar phenomenon. The comet, at that time, had a heliocentric distance of $2.44 \mathrm{AU}$, and a geocentric distance of $1.63 \mathrm{AU}$. The outburst of $17 \mathrm{P} /$ Holmes was exceptional: it was enormous in terms of its high brightness and spatial scale. The brightness of the comet increased from a total visual magnitude $m_{\mathrm{v}}=17$ to $m_{\mathrm{v}}=2.5$ (Green 2007). About 1\%-2\% of the nucleus was released during the phenomenon (Altenhoff et al. 2009; Schleicher et al. 2009; Sekanina 2008). Before the 2007 apparition, no other significant increase in magnitude had been reported since the comet discovery.

Comet outbursts are quite common but poorly understood phenomena. Only sometimes can they be associated with clear nucleus fragmentation, and many possible explanations have been proposed (Hughes 1991; Sekanina et al. 2002; Boehnhardt 2004). The causes of the outbursts in 17P/ Holmes remain unknown. A grazing collision by a small companion satellite of the comet has been proposed as the cause of the original discovery outburst (Whipple 1984; Whipple 1999), but the 2007 outburst argues against this interpretation. Following Altenhoff et al. (2009), the outburst may have been caused by water sublimation under a thick, air-tight dust mantle. Montalto et al. (2008) interpret their observations as an explosive event probably triggered by some internal instability process and not by an impact. Schleicher (2009) proposes that the explosive pressure inside the nucleus is caused by more volatile ices such as $\mathrm{CO}$ or $\mathrm{CO}_{2}$ vaporizing within a matrix of ice and dust; as a consequence of the phenomenon, fresh water ice has been exposed to direct solar heating.

Although the causes of this large outburst are uncertain, the event provided the opportunity to study the composition of $17 \mathrm{P} /$ Holmes. The composition class of the comet, as defined by A'Hearn et al. (1995) and Schleicher et al. (2007), is reported as "typical" (Schleicher 2009). In the first days after the outburst, the $\mathrm{H}_{2} \mathrm{O}$ production rate was very high compared to that of other comets at a similar heliocentric distance (Dello Russo et al. 2008; Schleicher 2009), as were the production rates of $\mathrm{C}_{2} \mathrm{H}_{6}$, $\mathrm{C}_{2} \mathrm{H}_{2}, \mathrm{HCN}$, and $\mathrm{CH}_{3} \mathrm{OH}$ with respect to $\mathrm{H}_{2} \mathrm{O}$. The exceptional outgassing detected during the outburst steeply decreased by a factor of 3 per day before decelerating yet further on October 28 . The overall gas production rate then declined by a factor of 7 between UT October 27.6 and November 2.3 (Dello Russo et al. 2008).

\section{Observation and data reduction}

Following this event, a request for director discretionary time was made to observe the comet $17 \mathrm{P} /$ Holmes with the high resolution spectrograph SARG (Gratton et al. 2001), mounted 
Table 1. Observing conditions.

\begin{tabular}{ccccccccc}
\hline \hline UT date & Image ID & $R$ & $\begin{array}{c}\text { Exposure time } \\
(\mathrm{s})\end{array}$ & $\begin{array}{c}\text { Spectral range } \\
(\mathrm{nm})\end{array}$ & $\begin{array}{c}r \\
(\mathrm{AU})\end{array}$ & $\begin{array}{c}\Delta \\
(\mathrm{AU})\end{array}$ & $\begin{array}{c}\dot{\Delta} \\
\left(\mathrm{km} \mathrm{s}^{-1}\right)\end{array}$ & $\begin{array}{c}\text { Projected slit length } \\
(\mathrm{km})\end{array}$ \\
\hline 22:33:18 2007 October 25 & JMEC0039 & 164000 & 180 & $462-792$ & 2.443 & 1.633 & -4.0099 & 4674.0 \\
22:59:24 2007 October 25 & JMEC0041 & 57000 & 600 & $360-514$ & 2.443 & 1.633 & -3.9427 & 4674.0 \\
21:33:39 2007 December 2 & JODF0017 & 29000 & 2700 & $462-792$ & 2.591 & 1.697 & 10.6591 & 9824.0 \\
22:24:48 2007 December 2 & JODF0018 & 29000 & 2700 & $360-514$ & 2.591 & 1.697 & 10.7384 & 9824.0 \\
\hline
\end{tabular}

Notes. Exposure starting time, spectrum identifier, resolving power, exposure time, spectral range covered by the spectrum, heliocentric and geocentric distances of the comet at the time of observation, geocentric velocity of the comet at the time of observation, and projected slit length on the comet.

on the $3.5 \mathrm{~m}$ Telescopio Nazionale Galileo (TNG) in the island of La Palma (Spain). The request was accepted, and the comet was observed on two nights, October 25 and December 2, 2007. Two spectra were acquired on the first night of observation, and two more on the second night. The observing conditions are reported in Table 1.

SARG is a cross-dispersed echelle spectrograph covering simultaneously the wavelength range between 3700 and $10000 \AA$; a selection of 6 slits and 4 grisms is available. The spectrum JMEC0039 was acquired with the yellow grism, covering the range 462-792 nm, while JMEC0041 was acquired with the blue grism, covering the range 360-514 nm. On December 2, both grisms were used (the yellow one for JODF0017 and the blue one for JODF0018). The resolving power varied between $R=29000$ (both spectra acquired on December 2) and $R=$ 164000 (spectra acquired on October 25), depending on the selected slit. At the time of observations, 17P/ Holmes heliocentric distance varied between 2.443 and $2.591 \mathrm{AU}$, while the geocentric distance varied between 1.633 and $1.697 \mathrm{AU}$. The variation in the projected slit length on the comet was between 4674 and $9824 \mathrm{~km}$.

The four spectra were reduced and analyzed following standard procedures, using the IRAF ECHELLE package. The data along the slit length were summed, obtaining one-dimensional spectra 55 orders each. ThAr lamp spectra were used for the wavelength calibration. By fitting the Thorium line positions for all the orders of each spectrum, dispersion solutions were achieved with rms errors generally lower than $10 \mathrm{~m}$. After the wavelength calibration, all spectra were Doppler shifted and divided by the continuum as a final step in the reduction process. Telluric emission lines were identified by using the lines list published by Osterbrock et al. (1996).

\section{Data analysis}

The four spectra reduced are of different quality. The signal-tonoise ratio is lower in JMEC0039 and JMEC0041 than in the spectra acquired on December 2. In all the four spectra, anyway, many emission lines can be detected.

High-resolution comet spectra in the optical range appear to be very crowded because they are formed mainly by molecular bands of various fragment species (Feldman et al. 2004). When a comet is approaching the Sun, $\mathrm{CN}$ emission is generally seen first, followed by species such as $\mathrm{C}_{3}, \mathrm{C}_{2}$, and $\mathrm{NH}_{2}$. When the comet is moving away, the emissions disappear in reverse order. The lines detected in the spectra that we analyzed are typical of comets observed at a distance from the Sun larger than $2 \mathrm{AU}$. Most of the emission lines detected can be attributed to $C_{2}$ and

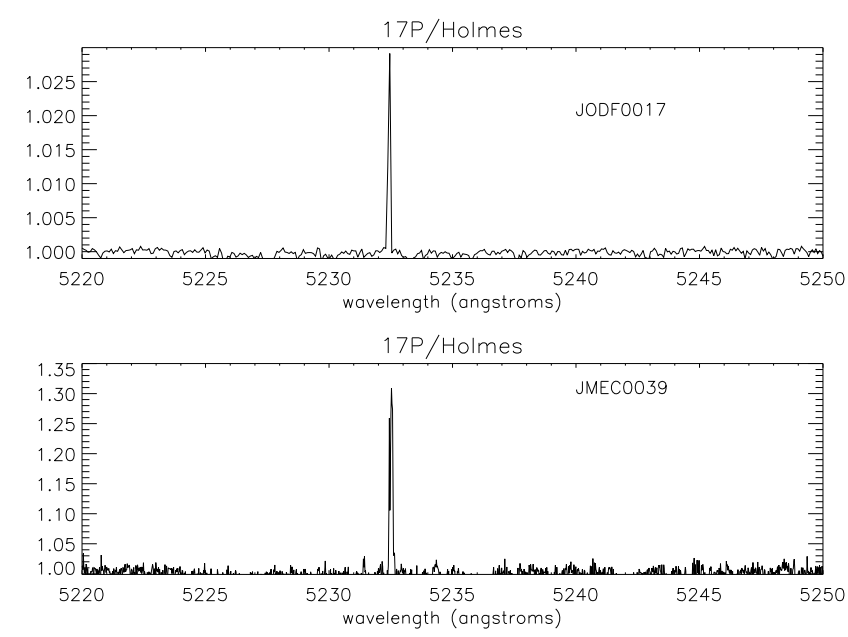

Fig. 1. The strong emission line at $5231.7 \AA$ that can be be attributed to $C_{2}$ (Swan band), visible in both spectra, is shown for JODF0017, acquired on December 2 (upper panel), and JMEC0039, acquired on October 25 (lower panel).

$\mathrm{NH}_{2}$. We now present a more detailed analysis of each of the species whose emission lines were detected and identified:

$\mathbf{C}_{2}$. At this distance from the Sun it is well known that $\mathrm{C}_{2}$ Swan-band emission lines can easily be detected in the coma of comets. In the spectra that we analyzed, we found many lines that can be attributed to this species, widespread across the entire spectral range. While many $\mathrm{C}_{2}$ lines overlap with $\mathrm{NH}_{2}$ lines, we found regions where only $\mathrm{C}_{2}$ is present and the identification is certain. The $\mathrm{C}_{2}$ lines are visible in the spectra of both observation nights (Fig. 1).

$\mathbf{N H}_{2}$. As for $\mathrm{C}_{2}, \mathrm{NH}_{2}$ lines are widespread in the optical range, and many lines that can be attributed to $\mathrm{NH}_{2}$ are detectable in the four analyzed spectra. Some of the lines can be attributed to $\mathrm{NH}_{2}$ alone, while other lines are found interspersed and blended with $\mathrm{C}_{2}$ lines.

CN. In the spectrum JODF0018, many CN lines are visible (Fig. 2), all belonging to the violet electronic band system. It must be noted that in the corresponding spectrum acquired on October 25 the blue part, where $\mathrm{CN}$ lines can be found, is featureless, probably because of the lower signal-to-noise ratio.

$\mathbf{C}_{3}$. A dozen $\mathrm{C}_{3}$ lines are detectable in the spectrum JODF0018, in the range 3982-4134 $\AA$.

CH. A few CH lines around $3900 \AA$ can be identified in the spectrum JODF0018 (Fig. 2). 


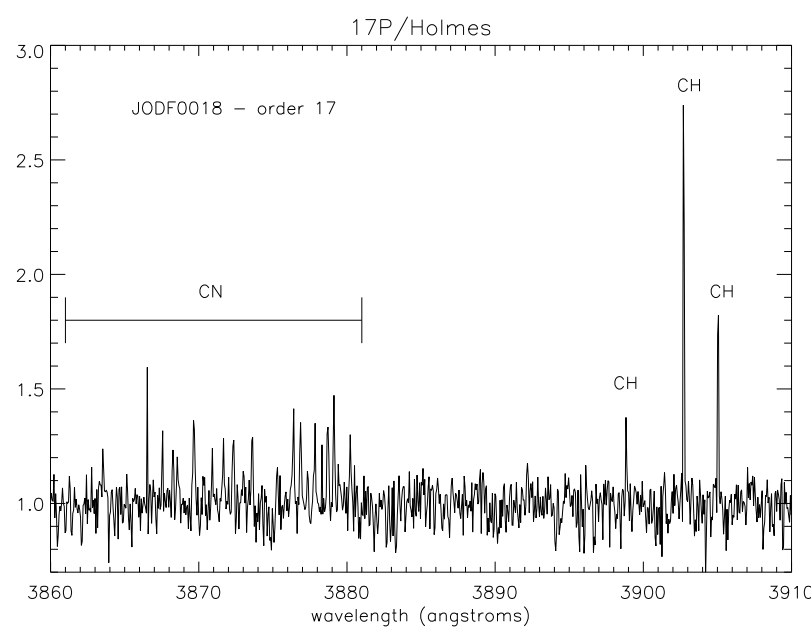

Fig. 2. Spectrum JODF0017, order 17. The emission lines visible in the plot can be attributed to $\mathrm{CN}$ and $\mathrm{CH}$, as labeled.

Unidentified lines. Many unidentified emission lines can be detected in the optical range, and consequently in the range covered by the four analyzed spectra. There are even regions (for example 6400-6800 ̊) in which they outnumber the identified lines. It is probable that most of the so-called "unidentified" lines can be attributed to not yet catalogued transitions of common species such as $\mathrm{C}_{2}$ and $\mathrm{NH}_{2}$, but the possibility that a "new" minor species is hidden between these lines cannot be discarded. This is the reason why the study of these lines is interesting. We catalogue them in the comets we have observed until now (Saba et al. 2005; Cremonese et al. 2007; Capria et al. 2008), looking for possible correlations with the heliocentric distance and other observation parameters, with the long-term aim of attempting or suggesting possible identifications. Many of the lines labeled as unidentified in the $17 \mathrm{P} /$ Holmes spectra are detectable in the corresponding spectra of the other comets we have observed at closer distances from the Sun, while some of them have not been previously detected.

\section{Analysis of the atomic oxygen emission lines}

In the optical region of cometary spectra, three forbidden $\mathrm{O}$ transitions are usually detectable, while they are not always separable from the corresponding telluric lines: the green line at $5577.339 \AA\left({ }^{1} \mathrm{~S}_{-}{ }^{1} \mathrm{D}\right)$ and the red doublet at $6300.304 \AA$ and $6363.776 \AA\left({ }^{1} \mathrm{D}-{ }^{3} \mathrm{P}\right)$ (Feldman et al. 2004; Cochran \& Cochran 2001; Cochran 2008). In the spectra JMEC0039, taken on October 25, and JODF0017, taken on December 2, some of these emission lines are visible. The green line is detectable in JMEC0039 and JODF0017, the red line at 6300.304 $\AA$ is detectable in both spectra and the red line at $6363.776 \AA$ is detectable only in JODF0017 (see Figs. 3 and 4).

The resolution necessary to separate the cometary $\mathrm{O}$ lines from the telluric O lines depends on the Doppler shift of the comet with respect to Earth, but high resolution is usually needed. The green line is particularly difficult to study, because this emission is found in the middle of the $C_{2}(1,2)$ P-branch.

The [OI] lines cannot be produced by solar resonance fluorescence excitation of the ground-state oxygen atom, but instead represent "prompt emission", which means that they can be produced directly in the excited states by photodissociation of the parent molecule. About $95 \%$ of oxygen atoms excited in the ${ }^{1} \mathrm{~S}$ state decay to the ground ${ }^{3} \mathrm{P}$ state via the ${ }^{1} \mathrm{D}$ state, while $5 \%$ of them decay directly to the ground state. If the green line is present, this implies that the red doublet is always present, while the opposite is not always true.

Many reactions can produce these forbidden oxygen lines (Bhardwaj \& Haider 2002), not all of which involve the water molecule: they may also involve $\mathrm{CO}, \mathrm{CO}_{2}$ and other minor species. The intensity ratio of the green to the sum of the two red lines can provide a clue to the identity of the parent molecule(s) of $\mathrm{O}$ atoms in the cometary coma. The intensity $\mathrm{I}(\mathrm{R})$ of an emission line can be written as (Festou \& Feldman 1981)

$I=10^{-6} \tau_{\mathrm{p}}^{-1} \alpha \beta N$,

where $\tau_{\mathrm{p}}$ is the dissociative lifetime of the parent, $\alpha$ the yield of photodissociation, $\beta$ the branching ratio, and $N$ the column density of the parent molecule. In the case of a red doublet, the ratio of the two line strengths should be the same as the ratio of their branching ratios, because they are both transitions from the $\left(2 p^{4}\right)^{1} \mathrm{D}$ state to the $\left(2 p^{4}\right)^{3} \mathrm{P}$ ground state.

The green to red intensity ratio can be written as (Cochran \& Cochran 2001)

$$
\frac{I_{5577}}{I_{6300}+I_{6364}}=\frac{\tau_{\text {green }}^{-1} \alpha_{\text {green }} N_{\text {green }} \beta_{5577}}{\tau_{\text {red }}^{-1} \alpha_{\text {red }} N_{\text {red }}\left(\beta_{6300}+\beta_{6364}\right)},
$$

and, if it is assumed that there is only one parent, the column densities are almost the same. The effective excitation ratio for dissociation of a parent molecule is proportional to $\tau^{-1} \alpha \beta$. Festou \& Feldman (1981) computed the theoretical value of this ratio for three different molecules, their results being 0.1 in the case of $\mathrm{H}_{2} \mathrm{O}$, and 1 in the case of $\mathrm{CO}_{2}$ and $\mathrm{CO}$. The results of Festou \& Feldman (1981) were questioned (Huestis \& Slanger 2006) on the basis that some of the photodissociation yields used in this computation may not be valid, but at the moment no more reliable values are available. It must be noted that the theoretical value of the intensity ratio of the two red lines is, as given by Storey \& Zeippen (2000), 3.027; this provides a way of testing the quality of the data when computing the intensity ratio of the green to the sum of the red lines.

In the spectrum JMEC0039, acquired on October 25, the green line and the red line at $6300.304 \AA$ are both detectable (Fig. 3). The red line at $6363.776 \AA$ cannot be resolved from the continuum. The green line is weak, and the contamination with the surrounding $C_{2}$ lines cannot be excluded. The red line is clearly visible and separable from the telluric line. We know that the red line at $6363.776 \AA$ must be present, even if it is too weak to be detectable. If we derive its value from the theoretical value of the ratio of the two red lines, we can make a rough estimate of the value of the green to the sum of the two red lines. The result is $0.1 \pm 0.11$, which implies that the water molecule is the main parent of the $\mathrm{O}$ emission detected in the spectrum JMEC0039.

For the observation performed on December 2, the situation is more favorable and the three atomic $\mathrm{O}$ lines are all detectable in the spectrum JODF0017 (see Fig. 4). The contamination of the green line by the $\mathrm{C}_{2}(1,2) \mathrm{P}$-branch, if any, seems to be very low. We computed the intensity ratio of the green line to the sum of the red lines for this spectrum, obtaining the result $0.3 \pm 0.1$. The intensity ratio of the two red lines is 3.25 , close to the theoretical value of 3.027 (Storey \& Zeippen 2000), so we can consider the value of the intensity ratio of the green line with respect to the red lines to be acceptable. 

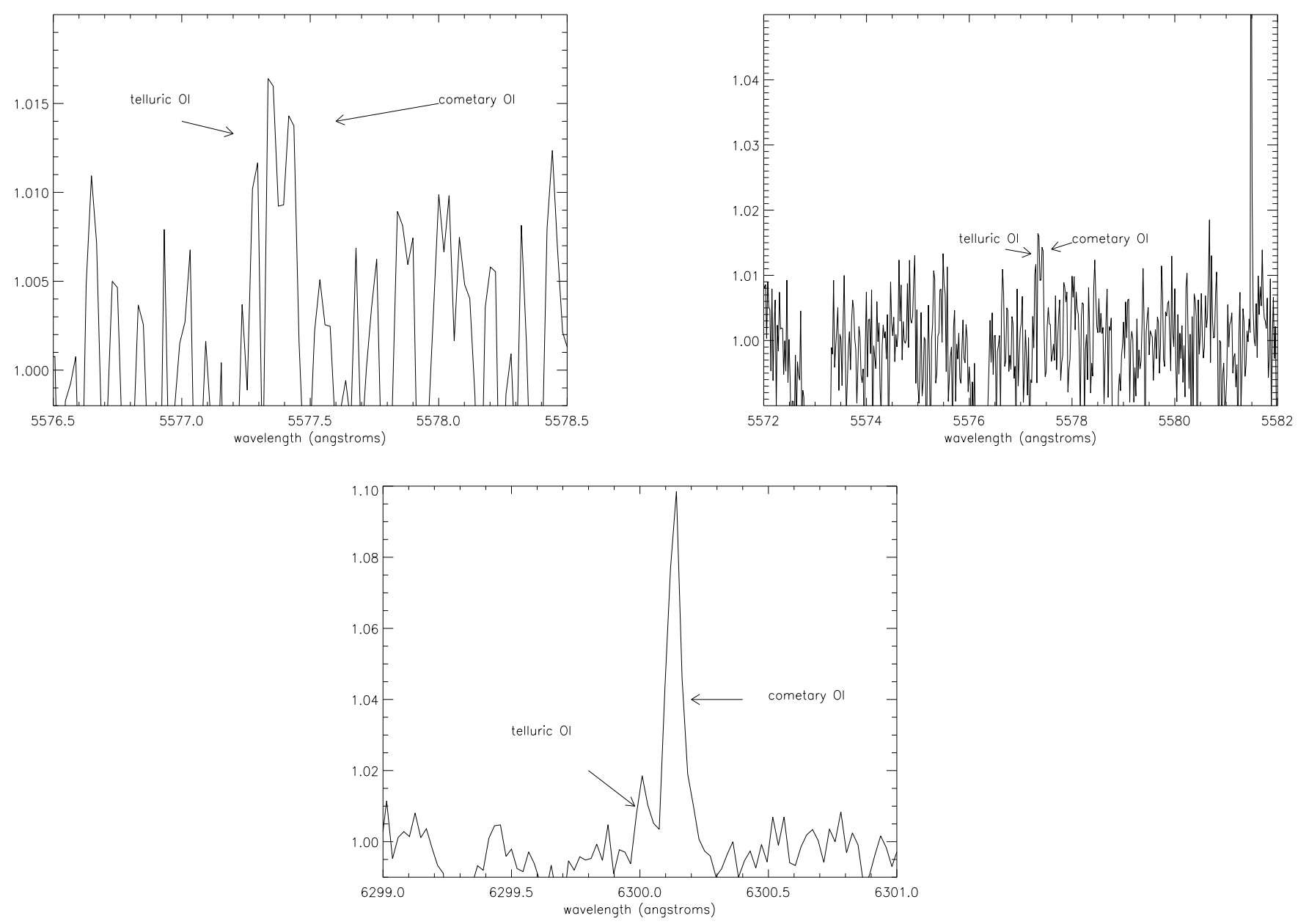

Fig. 3. Atomic oxygen lines for the first night of observation, October 25, 2007 (spectrum JMEC0039). The units of the vertical axis are arbitrary. On the higher left and right panels: green line at $5577.339 \AA$. All the other emission lines can be attributed to $\mathrm{C}_{2}$. On the lower panel: red line at $6300.304 \AA$.

\section{Discussion}

In the first days after the outburst, the $\mathrm{H}_{2} \mathrm{O}$ production rate was very high compared to other comets (Dello Russo et al. 2008; Schleicher 2009), as were the production rates for $\mathrm{C}_{2} \mathrm{H}_{6}, \mathrm{C}_{2} \mathrm{H}_{2}$, $\mathrm{HCN}$, and $\mathrm{CH}_{3} \mathrm{OH}$ with respect to $\mathrm{H}_{2} \mathrm{O}$. In all cases, we note that coma abundances in $17 \mathrm{P} /$ Holmes may not be directly comparable to coma abundances determined in other comets because most of the detailed chemical information about parent volatiles in comets is obtained at smaller heliocentric distances, typically close to $1 \mathrm{AU}$. The only exception would be the comet C/1995 O1 (Hale-Bopp), which was followed till large heliocentric distances, but Hale-Bopp, besides having an exceptionally large nucleus size, was a long-period comet, with an origin and a thermal history that differed from that of short-period comets. The exceptional outgassing detected during the outburst steeply decreased by a factor of 3 per day before slowing down on October 28 (Dello Russo et al. 2008). Biver et al. (2008), Schleicher (2009) and Drahus et al. (2008), who observed the comet at the beginning of December, agree that at that time the outgassing appeared to be what should be expected for a typical nucleus of that size at that heliocentric distance. It is clear that the two nights of our observation correspond to very different situations in terms of comet activity. Unfortunately, because of the poor quality of the spectra observed in October, and the spectra not having been calibrated in flux, a direct comparison between the results of the two nights is impossible.

It could be that this strong difference in the activity level of $17 \mathrm{P} /$ Holmes is instead reflected in the atomic $\mathrm{O}$ line intensity ratio as measured or estimated from our data. The intensity ratio of the green to red atomic $\mathrm{O}$ lines has been measured for few comets (see Table 2). Most of the comets listed in the table were observed while they were orbiting at heliocentric distances close to $1 \mathrm{AU}$, and the value of the ratio is around 0.1 , implying that the water molecule is the main parent of the emission lines. Only for the comets C/1995 O1 Hale-Bopp and 116P/Wild 4 was the ratio value higher. In the case of Hale-Bopp, a possible explanation (Bockelée-Morvan et al. 2004) is that CO abundance with respect to water was very high in this exceptional comet (around $12 \%$ at the nucleus, and 23\% in the coma extendedsource effect), thus its oxygen line ratio should tend towards unity. $116 \mathrm{P} /$ Wild 4 is the only other comet, observed at a heliocentric distance comparable to $17 \mathrm{P} /$ Holmes during the outburst event, for which an intensity ratio has been obtained (Furusho et al. 2006). At the moment of the observation, this comet was orbiting at $2.4 \mathrm{AU}$, and the intensity ratio derived by Furusho et al. (2006) was 0.155 (see Table 2). It is well known that at a distance as low as around 2.5 AU water sublimation may not be the main or only driver of cometary activity (Meech \& Svoren 2004), and the sublimation of more volatile molecules such as 

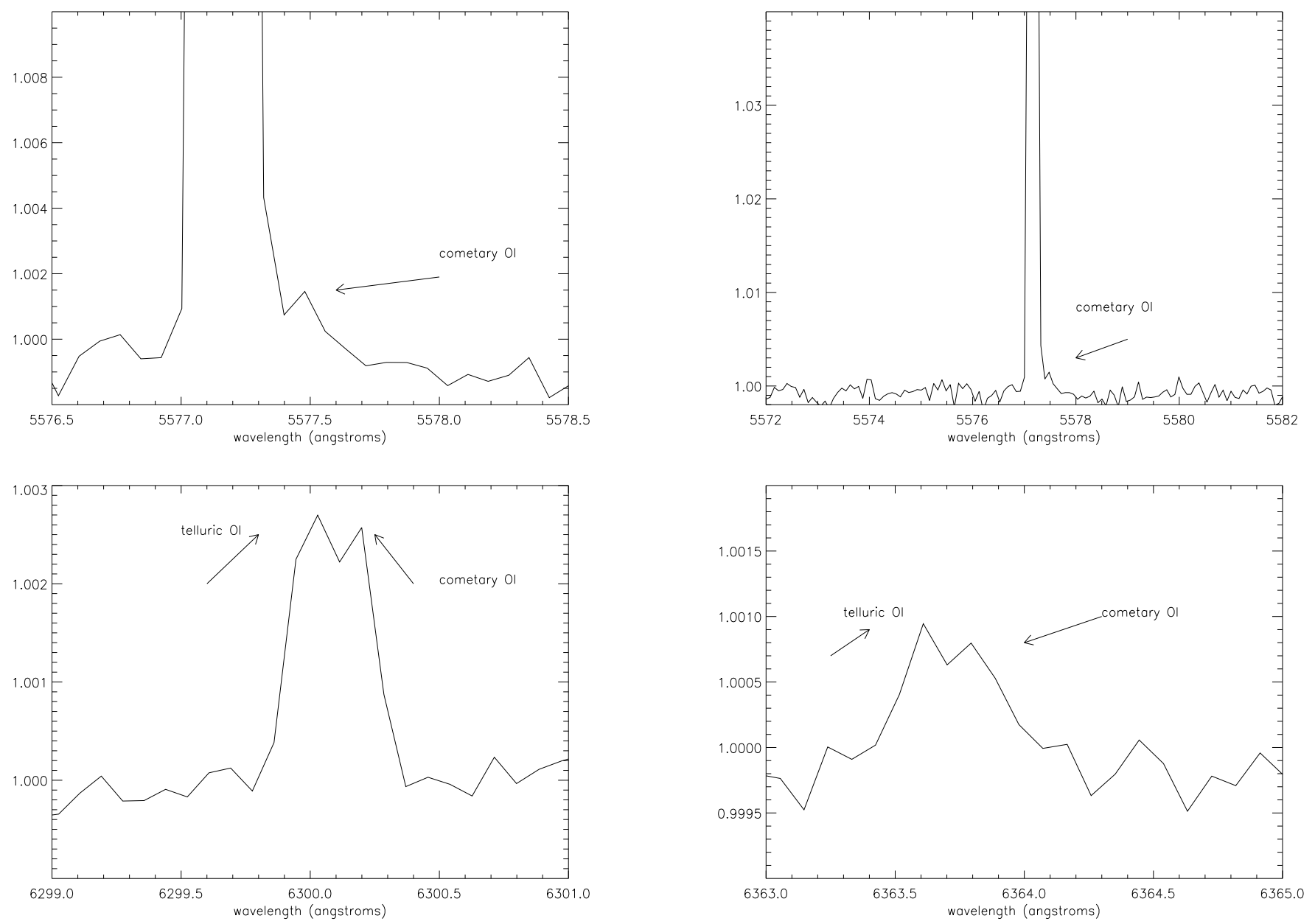

Fig. 4. Atomic O lines for the second night of observation, December 2, 2007 (spectrum JODF0017). The units of the vertical axis are arbitrary. On the higher left and right panels: green line at $5577.339 \AA$. On the lower panels: red line at $6300.304 \AA$ (left) and red line at $6363.776 \AA$ (right).

Table 2. Oxygen lines ratio in the literature.

\begin{tabular}{llll}
\hline \hline Comet $^{a}$ & $\frac{I_{5577}}{I_{6300+I_{6364}}}$ & $r(\mathrm{AU})^{b}$ & $\Delta(\mathrm{AU})^{c}$ \\
\hline C/Iras-Araki-Alcock (1) & $0.022-0.034$ & 1.02 & $\ldots$ \\
C/1996 B2 Hyakutake (2) & $0.12-0.15$ & $1.083-1.002$ & $0.120-0.119$ \\
C/1995 O1 Hale-Bopp (3) & $0.22-0.18$ & $0.920-0.991$ & $1.322-1.627$ \\
C/1999 S4 LINEAR (4) & $0.06 \pm 0.01$ & $0.97-0.79$ & $1.21-0.45$ \\
153P/Ikeya-Zhang (5) & $0.12 \pm 0.1$ & 0.89 & 0.43 \\
116P/Wild 4 (6) & $0.155 \pm 0.007$ & 2.4 & 0.43 \\
9P/Tempel 1 (7) & $0.056-0.13 \pm 0.1$ & 1.5 & 0.9 \\
17P/ Holmes (this work) & $0.3 \pm 0.1$ & 2.6 & 1.7 \\
\hline
\end{tabular}

(a) References: (1) Cochran (1984); (2) Morrison et al. (1997); (3) Zhang et al. (2001); (4) Cochran \& Cochran (2001); (5) Capria et al. (2005); (6) Furusho et al. (2006); (7) Capria et al. (2008). ${ }^{(b)}$ Heliocentric distance. ${ }^{(c)}$ Geocentric distance.

$\mathrm{CO}$ and $\mathrm{CO}_{2}$ is thought to usually give a strong contribution to the observed gas production. It is straightforward to attribute this value, and the one obtained by us for the comet $17 \mathrm{P} /$ Holmes, to in this case a strong contribution of $\mathrm{CO}$ or $\mathrm{CO}_{2}$ (or both of them), and $\mathrm{H}_{2} \mathrm{O}$ not being the only parent of the emission lines.

We must now explain why the estimated intensity ratio on October 25 appeared to be slightly different, implying that water molecules were the main parent. During the outburst, the water production rate was very high (Dello Russo et al. 2008;
Schleicher 2009), surely much higher than the usual production rate for comets at that heliocentric distance, and typical instead of comets orbiting close to $1 \mathrm{AU}$ from the Sun. The intensity ratio estimated by us may reflect a situation in which, notwithstanding the large heliocentric distance, the $\mathrm{H}_{2} \mathrm{O}$ production rate is so high that water molecules are the main parent of atomic $\mathrm{O}$ emission lines.

We note that Schleicher (2009) hypothesized that the sublimation of gases more volatile than water could be the origin of 
the recurrent outbursts of $17 \mathrm{P} /$ Holmes. We previously discussed the possibility of comet activity sustained by $\mathrm{CO}$ and $\mathrm{CO}_{2}$ in the case of comets far from the Sun, when the sublimation of water ice cannot take place at a level high enough to justify strong activity (Capria et al. 2009). We consider this hypothesis to be a real possibility and a viable explanation of the observed phenomena. The devolatilized mantle, blown up by the explosion of gas pockets in which the pressure builds up, is typical of this kind of scenario, and can easily explain the observed dust behavior. It is only necessary to assume that at the interior of the nucleus volatile molecules survive, as either ices or gases trapped in the amorphous ice matrix. This is not impossible, given the low thermal conductivity coupled with very low internal temperatures. If some volatile-rich zones were to exist in the nucleus beneath a thick devolatilized dust mantle, the pressure could build-up, even periodically, to produce an explosion (outburst). The explosion would then trigger an enormous amount of water sublimation, which would account for the intensity ratio obtained for $17 \mathrm{P} / \mathrm{P}$ Holmes while it was orbiting at 2.4 AU from the Sun.

\section{Conclusion}

We have analyzed data of comet 17P/ Holmes acquired with the high resolution echelle spectrograph SARG at TNG for two nights, on October 25, 2007, immediately after a major outburst event, and on December 2, 2007, one month after. Four high resolution visible spectra, two for each night of observation, were analyzed. In one of the spectra acquired on December 2, the three atomic oxygen lines were detected and their intensity ratio derived, giving a result of $0.3 \pm 0.1$. This is the highest value measured until now for this ratio, being indicative, following Festou \& Feldman (1981), of parent molecules that were not only (or not mainly) $\mathrm{H}_{2} \mathrm{O}$, but had a strong contribution from a different species, probably $\mathrm{CO}$ and/or $\mathrm{CO}_{2}$.

In one of the spectra acquired on October 25, the green line and one of the red doublet lines were detected. This gave us the possibility of estimating a value for the intensity ratio, which is $0.1 \pm 0.11$. In this case the intensity ratio implies that $\mathrm{H}_{2} \mathrm{O}$ is the main parent of the observed emission lines. We have interpreted the different values obtained for the two observing nights by assuming that in the days during the outburst the water production rate was exceptional, more typical of a comet orbiting close to $1 \mathrm{AU}$ than of a comet at this distance from the Sun. One month after, instead, 17P/ Holmes had returned to a behavior typical of a comet at $2.6 \mathrm{AU}$, at which distance $\mathrm{CO}$ and $\mathrm{CO}_{2}$ are understood to strongly contribute to sustain the activity.
Acknowledgements. This work has been supported by ASI-INAF contract no. I/015/07/0 "Solar System Exploration". Observations at TNG have been partially funded by the Fundación Galileo Galilei of the INAF (Istituto Nazionale di Astrofisica). We would like to thank the director of TNG, E. Oliva, and the TAC for granting us discretional director time, and the staff of TNG, and F. Ghinassi in particular, for performing the observations.

\section{References}

A'Hearn, M. F., Millis, R. L., Schleicher, D. G., et al. 1995, Icarus, 118, 223 Altenhoff, W. J., Kreysa, E., Menten, K. M., et al. 2009, A\&A, 495, 975 Bhardwaj, A., \& Haider, S. A. 2002, Adv. Space Res., 29(5), 745 Biver, N., Bockelée-Morvan, D., Wiesemeyer, H., et al. 2008, ACM

Bockelée-Morvan, D., Crovisier, J., Mumma, M. J., \& Weaver, H. A. 2004, The composition of cometary volatiles. In Comets II, ed. M.C. Festou, U. Keller, \& H. A. Weaver (Tucson: Univ. Arizona Press)

Boehnhardt, H. 2004, Split comets. In Comets II, ed. M.C. Festou, U. Keller, \& H. A. Weaver (Tucson: Univ. Arizona Press)

Capria, M. T., Cremonese, G., Bhardwaj, A., et al. 2005, A\&A, 442, 1121 Capria, M. T., Cremonese, G., Bhardwaj, A., et al. 2008, A\&A, 479, 257 Capria, M. T., Coradini, A., De Sanctis, M. C., et al. 2009, A\&A, 504, 249 Cochran, A. L. 2008, Icarus, 198, 181

Cochran, A. L., \& Cochran, W. D. 2001, Icarus, 154, 381

Cochran, W. D. 1984, Icarus, 58, 440

Cremonese, G., Capria, M. T., \& De Sanctis, M. C. 2007, A\&A, 461, 789 Drahus, M., Paganini, L., Ziurys, L. M., et al. 2008, ACM

Dello Russo, N., Vervack, R. J., Weaver, H. A., et al. 2008, ApJ, 680, 793

Feldman, P. D., Cochran, A. L., \& Combi, M. R. 2004, Spectroscopic investigation of fragment species in the coma. In Comets II, ed. M. C. Festou, U. Keller, \& H. A. Weaver, Univ. (Tucson: Arizona Press)

Festou, M. C., \& Feldman, P. D. 1981, A\&A, 103, 154

Furusho, R., Kawakita, H., Fuse, T., et al. 2006, Adv. Space Res., 38, 2006

Gratton, R. G., Bonanno, G., Bruno, P., et al. 2001, Exp. Astron., 12, 107

Green, D. W. E. 2007, IAU Circ. 8886

Huestis, D. L., \& Slanger, T. G. 2006, DPS $\sharp 38, \sharp 62.20$, Amer. Astron. Soc.

Hughes, D. W. 1991, Possible mechanisms for cometary outbursts, in Comets in the post-Halley era, ed. Jr. Newburn, M. Neugebauer, \& J. Rahe (Dordrecht: Kluwer Academic Publisher)

Meech, K. J., \& Svoren, J. 2004, Using cometary activity to trace the physical and chemical evolution of cometary nuclei, in Comets II, ed. M. C. Festou, U. Keller, \& H. A. Weaver (Tucson: Univ. Arizona Press)

Montalto, M., Riffeser, A., Hopp, U., et al. 2008, A\&A, 479, L45

Morrison, N. D., Knauth, D. C., Mulliss, C. L., \& Lee, W. 1997 PASP, 109, 676

Osterbrock, D. E., Fulbright, J. P., Martel, A. L., et al. 1996, PASP, 108, 277 Saba, L., Capria, M. T., \& Cremonese, G. 2005, Mem. Soc. Astron. Ital. Suppl.,
6, 137

Schleicher, D. G. 2009, ApJ, 138, 1062

Schleicher, D. G., Farnham, T. L., \& Bair, A. N. 2007, in Workshop on Planetary Atmospheres, LPI Contribution No. 1376, 103

Sekanina, Z. 2008, Int. Comet Q., 30, 3

Sekanina, Z., Jehin, E., \& Boehnhardt, H. 2002, ApJ, 572, 679

Storey, P. J., \& Zeippen, C. J. 2000, MNRAS, 312, 813

Whipple, F. 1984, Icarus, 60, 522

Whipple, F. 1999, Plan. Space Sci., 47, 301

Zhang, H. W., Zhao, G., \& Hu, J. Y. 2001, A\&A, 367, 1049 